\title{
ON ONE-SIDED DIVISION INFINITE-DIMENSIONAL NORMED REAL ALGEBRAS
}

\author{
José Antonio Cuenca Mira
}

Dedicated to the memory of Pere Menal

\begin{abstract}
In this note we introduce the concept of Cayley homomorphism which is closely related with those of composition algebra and normalized orthogonal multiplication. The key result shows the existence of certain types of Cayley homomorphisms for infinite dimension. As an application we prove the existence of left division infinite-dimensional complete normed real algebras with left unity.
\end{abstract}

Let $K$ be a field of characteristic different from $2, V$ and $W$ vector $K$-spaces (not necessarily with finite dimension) everyone endowed with a nondegenerate symmetric bilinear form. These forms will be denoted by $\langle\mid\rangle$. We shall say that $(V,-, e)$ is a Cayley triad (over $V$ ) if - is an involutive isometry of $V$ and $e$ an element in $V$ such that $\bar{e}=e$. We denote by $\mathcal{L}(W)$ the vector subspace of the linear maps $T$ in $\operatorname{End}_{K}(W)$ which have an adjoint map $T^{*}$ with respect to $\langle\mid\rangle$. The linear map $S$ : $V \rightarrow \mathcal{L}(W)$ carrying every $x$ to $S_{x}$ is said to be a Cayley homomorphism (briefly, homomorphism) from the Cayley triad to $W$ if the following conditions are satisfied:

1) $S_{\bar{x}^{0}} S_{x}=\langle x \mid x\rangle$ Id for any $x$ in $V$ (where Id denotes the identity operator on $W)$.

2) $S_{x}^{*}=S_{x}$.

3) $S_{e}=$ Id.

It is easy to show that 2) is equivalent to the following condition:

2) For any $x, y, z$ in $V$ we have $\left\langle S_{x}(y) \mid S_{x}(z)\right\rangle=\langle x \mid x\rangle\langle y \mid z\rangle$.

Linearizing equality 1) we obtain

$$
\left.S_{x^{0}} S_{y}+S_{\bar{y}^{0}} S_{x}=2\langle x \mid y\rangle\right) \mathrm{Id}
$$


for any $x, y$ in $V$. On the other hand 1),2) and 3) yield to $\langle e \mid e\rangle=1$.

Assume that $V=W$ is a composition $K$-algebra whose symmetric bilinear form is $\langle\|\rangle$ and with Cayley antiautomorphism -. If $e$ is the unity of $V$ then the map sending every element $x$ in $V$ to the rigth multiplication operator $R_{x}$ is a Cayley homomorphism from the Cayley triad $(V,-, e)$ to $V$. Conversely, let $V$ be a finitc-dimensional vector space endowed with a nondegenerate symmetric bilinear form, assume $(V,-, e)$ a Cayley triad over $V$ and $S: V \rightarrow \mathcal{L}(V)=\mathrm{End}_{K}(V)$ a Caylcy homomorphism from the triad to $V$. As in $[5$, proof of Proposition $I$ in p. 25], we can define a product on $V$ for which $V$ becomes a composition algebra. Cayley homomorphisms are also closely related with normalized orthogonal multiplications [1, p. 140-158].

Theorem 1. Let $K$ be an ordered field where every positive element has a square root, $H$ a vector $K$-space with countable infinite dimension which is endowed with a positive definite symmetric bilinear form. Then there are a Cayley triad $(H,-, e)$ over $H$ and an homomorphism from this triad to $H$. Moreover - only fixes the vector line spanned by $e$.

Proof: It is well known that $H$ has an orthonormal basis $e_{0}, e_{1}, \ldots, e_{n}, \ldots$ (see for instance $[2$, Theorern 29]). For any integer $n \geq 0$ let $V_{n}$ (resp. $W_{n}$ ) be the subspace of $H$ spanned by the $n+1$ (resp. $2^{n}$ ) first elements of this basis. Let $e=e_{0}$. Denote by - the linear operator on $H$ fixing $e$ and sending the remainders elements of this basis into their opposites. For each $n$ the map - can be restricted to an involutive automorphism of $V_{n}$ which we shall also denote in the same way. Fvery $\left(V_{n},-, e\right)$ is a Cayley triad. We shall prove by induction that for every $n$ there exists a Cayley homomorphism $S^{(n)}$ from this triad to $W_{n}$ which satisfies the following property: for all $m<n$ and any $x \in V_{m}, y \in W_{m}$ we have $S_{x}^{(n)}(y)=S_{x}^{(m)}(y)$. Obviously the statement is true for $n=0$. Assume now that there exists an homomorphism $S^{(n)}$ from $\left(V_{n},-, e\right)$ to $W_{n}$ satisfying the required property. We shall define $S^{(n+1)}$. For this, firstly we observe that there exists an isometry $b_{n}$ from $W_{n}$ onto the orthogonal subspace of $W_{n}$ relative to $W_{n+1}$. So every element in $W_{n+1}$ can be written in a unique way as $x+b_{n}(y)$ with $x, y \in W_{n}$. An arbitrary element in $V_{n+1}$ is a sum $u+\lambda e_{n+1}$ with $u \in V_{n}$ and $\lambda \in K$. We denote by $S_{u+\lambda e_{n+1}}^{(n+1)}$ the linear map from $V_{n+1}$ to $W_{n+1}$ defined in the following way

$$
S_{u+\lambda e_{n+1}}^{(n+1)}\left(x+b_{n}(y)\right)=S_{u t}^{(n)}(x)-\lambda y+b_{n}\left(S_{\tilde{u}}^{(n)}(y)+\lambda x\right) .
$$

The $\operatorname{map} S^{(n+1)}: V_{n+1} \rightarrow \mathcal{L}\left(W_{n+1}\right)=\operatorname{End}_{K}\left(W_{n+1}\right)$ given by $u+\lambda e_{n+1} \rightarrow S_{u+\lambda e_{n+1}}^{(n+1)}$ is an homomorphism from the Cayley triad 
$\left(V_{n+1},-, e\right)$ to $W_{n+1}$ which satisfies the required property. For any elements $x, y \in H$ there cxist a $V_{n}$ such that $x, y \in V_{n}$. The element $S_{x}^{(n)}(y)$ does not depend of the chosen vector space $V_{n}$ and will be denoted by $S_{x}(y)$. Every $S_{x}$ is a linear operator on $H$ which has adjoint operator equal to $S_{\bar{x}}$ and the map $x \rightarrow S_{x}$ from $H$ to $\mathcal{L}(H)$ is an homomorphism from the Cayley triad $(H,-, e)$ to $H$.

Theorem 2. Let $H$ be an infinite-dimensional separable real Hilbert space. Then there exist a Coyley triad $(H,-, e)$ and an homomorphism from this triad to $H$.

Proof: There exists in $\mathrm{H}$ a complete orthonormal system of countable infinite cardinal. Let $H^{\prime}$ be the vector subspace spanned by this set. By theorem 1, there are a. Cayley triad $\left(H^{\prime},-, e\right)$ and an homomorphism $S^{\prime}$ from this triad to $H^{\prime}$. We also denote by - the continuous extension of - to $H$. If $x, y \in H$ and $\left\{x_{n}\right\},\left\{y_{n}\right\}$ are sequences of elements in $H^{\prime}$ such that $x=\lim _{n \rightarrow \infty} x_{n}, y=\lim _{n \rightarrow \infty} y_{n}$, then we have that $\left\{S_{x_{n}}^{\prime}\left(y_{n}\right)\right\}$ is a Cauchy sequence in $H$. The limit does not depend of the chosen Cauchy sequences and is denoted by $S_{x}(y)$. For any $x, y \in H$ we have $\left\|S_{x}(y)\right\|=\|x\|\|y\|$. Moreover all $S_{x}$ is continuous with adjoint map $S_{x}$. So the linear map $S: H \rightarrow \mathcal{L}(H)$ given by $S: x \rightarrow S_{x}$ is an homomorphism from the Cayley triad $(H,-, e)$ to $H$.

We recall that a (nonassociative) algebra, $V$ is a left division algebra if for any nonzero element $x$ in $V$ the left multiplication operator $L_{x}$ is inversible. In a similar way right division algebras can be defined. The real algebra $V$ is said to be an absolute valued algebra if it is normed and it satisfies $\|x y\|=\|x\|\|y\|$ for any $x, y \in V$. If $H$ is a real Hilbert space, $(H,-, e)$ a Cayley triad over $H$ and $S$ a Cayley homomorphism from the triad to $H$, then $H$ with the product defined by $x y=S_{x}(y)$ becomes a left division algebra with left unity $e$ which is absolutc valued. So we have

Theorem 2'. In every separable real Hilbert space with infinite dimension can be defined a (nonassociative) product with which $H$ becomes a left division absolute valued algebra with left unity.

Remark 1. Theorem 2' shows the existence of real left division complete normed algebras with infinite dimension. It was conjectured by F. B. Wrigth [4] that every normed division algebra over the reals is finite-dimensional.

Remark 2. Let $\mathcal{M}$ be a set, $\mathcal{U}$ an ultrafilter on $\mathcal{M}$ and $\left\{H_{\gamma}\right\}_{\gamma \in \mathcal{M}}$ a family of real Hilbert spaces which are left division absolute valued 
algebras with left unity. Let $H$ be the $l^{\infty}$-sum of this family and $N$ the ideal of the elements $\left(x_{\gamma}\right)_{\gamma \in \mathcal{M}}$ such that $\lim _{u}\left\|x_{\gamma}\right\|=0$. Then $N$ is closed and $H / N$ is a normed algebra which will be denoted by $\left(H_{\gamma}\right) u$ and called the normed ultraproduct of $\left\{H_{\gamma}\right\}$ with respect to $\mathcal{U}$ (see [6]). Moreover for any element $\left[\left(x_{\gamma}\right)\right]$ in the ultraproduct we have

$$
\left\|\left[\left(x_{\gamma}\right)\right]\right\|=\lim _{u}\left\|x_{\gamma}\right\| .
$$

So this ultraproduct is a Hilbert space. It is easy to show that $\left(H_{\gamma}\right)_{t}$ is a left division absolute valued algebra with left unity. We can obtain from this that there exist real Hilbert spaces with infinite Hilbert dimension big enough over which we can define a product endowing it with a structure of left division absolute valued algebra with left unity. Finally we observe that A. Rodriguez [3] has given the structure theory of left division absolute valued algebras with left unity.

\section{References}

1. D. Husemoller, "Fibre bundles," Springer-Verlag, New York, second edition, 1975.

2. I. KAPLANSKY, "Linear algebra and geometry:" Chelsea, New York, 1974.

3. A. Rodríguez, Onc-sided division absolute valued algebras, to appear.

4. F. B. Wright, Absolute valued algebras, Proc. N.A.S. 39 (1953), $330-332$.

5. K. A. Zhevlakov, A. M. Suin'ko, I. P. Shestakov, A. I. ShrrSHOV, "Rings that are nearly associative," Academic Press, New York, 1982.

6. H. EinRICH, Ultraproducts in Banach space theory, J. Reine Angew. Math. 313 (1980), 72- 104.

Departamento de Álgebra, Geonetría y Topología

Universidad de Málaga

Apartado 59

29080 Málaga

SPAIN 